\title{
Antibiotic prescribing in mental health units across the Veterans' Health Administration: How much and how appropriate?
}

\author{
Jay J. Tieri PharmD ${ }^{1}$, Bruce Alexander BSc, PharmD ${ }^{1}$, Jason A. Egge PharmD, BCPS ${ }^{1}$, \\ Brett H. Heintz PharmD, BCPS-ID, AAHIVE ${ }^{1}$ and Daniel J. Livorsi MD, MSc ${ }^{1,2}$ (D) \\ ${ }^{1}$ Iowa City VA Health Care System, lowa City, lowa and ${ }^{2}$ Division of Infectious Diseases, Carver College of Medicine, University of Iowa, Iowa City, Iowa
}

\begin{abstract}
We evaluated antibiotic-prescribing across 111 mental health units in the Veterans' Health Administration. We found that accurate diagnosis of urinary tract infections is a major area for improvement. Because non-mental-health clinicians were involved in most antibiotic-prescribing decisions, stewardship interventions for mental health patients should have a broad target audience to be effective.
\end{abstract}

(Received 2 July 2021; accepted 16 September 2021; electronically published 21 October 2021)

Even though all US hospitals are required to have an antibiotic stewardship program, standard stewardship metrics like the standardized antimicrobial administration ratio do not capture antibiotic use in a hospital's mental health units. ${ }^{1}$ It is unclear how frequently antibiotics are used in these settings and whether there are opportunities to improve antibiotic-prescribing.

The objective of this study was to describe and evaluate antibiotic use across inpatient mental health units in an integrated healthcare network known as the Veterans' Health Administration (VHA).

\section{Methods}

The Institutional Review Board of the University of Iowa and the Research \& Development Committee of the Iowa City Veterans' Affairs Health Care System approved this study and waived informed consent.

Using the Veterans' Affairs Informatics and Computing Infrastructure (VINCI), we collected administrative data on all patients admitted to an inpatient mental health ward in VHA between January 1, 2016 and December 31, 2018. This cohort included data from inpatient mental health units across 111 unique medical centers, including 105 medical centers with an acute-care hospital. We excluded substance abuse rehabilitation units and domiciliaries.

Inpatient and postdischarge antibiotics were collected from the bar-coded medication administration records (BCMA) and outpatient medication files, respectively. Antibiotics included all agents and routes of administration listed in the National Healthcare Safety Network's (NHSN) Antimicrobial Use protocol. ${ }^{1}$ For each

Author for correspondence: Daniel Livorsi, E-mail: daniel-livorsi@uiowa.edu PREVIOUS PRESENTATION. This work was presented at the Iowa Pharmacy Association's Innovation and Research Forum on June 2-3, 2021, in Des Moines, Iowa.

Cite this article: Tieri JJ, et al. (2023). Antibiotic prescribing in mental health units across the Veterans' Health Administration: How much and how appropriate?. Infection Control \& Hospital Epidemiology, 44: 308-311, https://doi.org/10.1017/ ice. 2021.432 unique facility, antibiotic days of therapy (DOT) and days present were aggregated based on NHSN methodology.

Manual chart reviews were performed in a subset of the above cohort to assess adherence to standard recommended practices and professional guidelines for cystitis, or urinary tract infections (UTI), skin and soft-tissue infections (SSTIs), and acute respiratory tract infections (ARIs). Admissions with a qualifying ICD-10 code (Supplementary Table 1 online) were eligible for inclusion. For each infection type, we randomly selected an eligible admission from each facility. If a patient admission met our exclusion criteria (Supplementary Table 2 online), we continued to randomly sample eligible admissions from that facility until a case could be fully adjudicated using our criteria. At some sites a qualifying case could not be found. For each case adjudication, assessments were based on established algorithms (Supplementary Figs. 1-7). ${ }^{2,3}$ The primary review was performed by a postgraduate year 1 pharmacy resident (J.T.). An infectious disease (ID)-trained clinician (D.L. or B.H.) performed a secondary review of all included cases. We monitored the number of cases in which the ID-trained clinician changed the assessment.

\section{Results}

\section{Antibiotic use}

There were 252,588 patient admissions across the 111 mental health units. Mental health units averaged 759 admissions per year (SD, 436). During the study period, 27,401 (10.9\%) patient admissions had antibiotics administered while hospitalized on the mental health unit (Table 1). The diagnoses that were most commonly associated with an antibiotic prescription were UTI $(n=5,026,18.3 \%)$, SSTI ( $=2,992,10.9 \%)$, and ARIs $(\mathrm{n}=1,935,7.1 \%)$, which included acute bronchitis, pharyngitis, sinusitis and upper respiratory tract infections. The median total duration of therapy in patients who received antibiotics, including inpatient and after discharge, was 7 days (IQR 4-10).

Across all 111 sites, the median inpatient antibiotic DOT per 1,000 days present was 73.5 (IQR, 60.4-86.1) The most commonly

(c) The Author(s), 2021. Published by Cambridge University Press on behalf of The Society for Healthcare Epidemiology of America.. This is an Open Access article, distributed under the terms of the Creative Commons Attribution licence (http://creativecommons.org/licenses/by/4.0/), which permits unrestricted re-use, distribution, and reproduction in any medium, provided the original work is properly cited. 
Table 1. Characteristics of 252,588 Patient Admissions to Mental Health Units in the Veterans' Health Administration, 2016-2018

\begin{tabular}{|c|c|c|c|}
\hline Characteristic & $\begin{array}{l}\text { Antibiotic Received } \\
\qquad(n=27,401)\end{array}$ & $\begin{array}{l}\text { No antibiotic Received } \\
\qquad(\mathrm{n}=225,187)\end{array}$ & $\begin{array}{c}\text { Total } \\
(\mathrm{n}=252,588)\end{array}$ \\
\hline Age, mean y (SD) & $54.7(14.2)$ & $50.8(14.3)$ & $51.2(14.4)$ \\
\hline \multicolumn{4}{|l|}{ Sex, no. (\%) } \\
\hline Male & $23,168(84.5)$ & $204,307(90.7)$ & $227,475(90.1)$ \\
\hline Female & $4,233(15.5)$ & $20,880(9.3)$ & $25,113(9.9)$ \\
\hline \multicolumn{4}{|l|}{ Race, no. (\%) } \\
\hline White & $17,863(65.2)$ & $145,126(64.4)$ & $162,989(64.5)$ \\
\hline Black or African American & $7,646(27.9)$ & $63,225(28.1)$ & $70,871(28.0)$ \\
\hline American Indian or Alaska Native & $272(1.0)$ & $2,002(0.9)$ & $2,274(0.9)$ \\
\hline Native Hawaiian or Pacific Islander & $173(0.6)$ & $1,469(0.7)$ & $1,642(0.7)$ \\
\hline Asian & $115(0.4)$ & $1,404(0.6)$ & $1,519(0.6)$ \\
\hline Missing & $1,332(4.9)$ & $11,961(5.3)$ & $13,293(5.3)$ \\
\hline \multicolumn{4}{|l|}{ Ethnicity, no. (\%) } \\
\hline Hispanic or Latino & $1,295(4.7)$ & $13,623(6.0)$ & $14,918(5.9)$ \\
\hline \multicolumn{4}{|l|}{ Psychiatric diagnosis, no. (\%) } \\
\hline Substance use disorder & $19,920(72.7)$ & $173,123(67.8)$ & $193,043(76.4)$ \\
\hline Schizoaffective disorder & $17,284(63.1)$ & $143,330(63.6)$ & $160,614(63.6)$ \\
\hline Anxiety disorder & $14,545(53.1)$ & $128,240(56.9)$ & $142,785(56.5)$ \\
\hline Psychosis & $6,903(25.2)$ & $47,313(21.0)$ & $54,216(21.5)$ \\
\hline Personality disorder & $4,224(15.4)$ & $33,103(14.7)$ & $37,327(14.8)$ \\
\hline \multicolumn{4}{|l|}{ Comorbid conditions, no. (\%) } \\
\hline Drug abuse & $14,682(53.6)$ & $121,648(54.0)$ & $136,330(54.0)$ \\
\hline Alcohol use disorder & $13,434(49.0)$ & $120,291(53.4)$ & $133,725(52.9)$ \\
\hline Asthma/COPD & $6,478(23.6)$ & $38,507(17.1)$ & $44,985(17.8)$ \\
\hline Diabetes & $6,299(23.0)$ & $38,279(17.0)$ & $44,578(17.6)$ \\
\hline Neurologic disorder & $3,544(12.9)$ & $19,294(8.6)$ & $22,838(9.0)$ \\
\hline Dementia & $2,773(10.1)$ & $11,551(5.1)$ & $14,324(5.7)$ \\
\hline Renal failure & $2,044(7.5)$ & $10,339(4.6)$ & $12,383(4.9)$ \\
\hline Length of stay, median d (IQR) & $7(3-13)$ & $5(3-9)$ & $5(3-9)$ \\
\hline
\end{tabular}

prescribed agents, as quantified by DOT per 1,000 days-present, were trimethoprim-sulfamethoxazole (10.7), cephalosporins (9.5), tetracyclines (8.8), anti-influenza medications (8.3) and fluoroquinolones (8.0).

\section{Manual chart reviews to assess guideline-concordance}

Table 2 shows the findings from the manual chart reviews, including the percentage of cases in which the reviewers disagreed. Among 111 UTI cases, 27 cases $(24.3 \%)$ had documentation of altered mental status. For 56 cases (50.5\%), documentation indicated the absence of local genitourinary symptoms, and an additional 7 cases (6.3\%) had negative urine cultures collected before antibiotics were initiated. Therefore, the diagnosis of UTI was not supported by history or laboratory findings in 63 cases (56.8\%). In 21 cases (18.9\%) documentation could not be found that indicated that anyone had asked the patient about local genitourinary symptoms.

For 101 SSTI cases, a documented history of active intravenous drug use was present in 25 cases (24.8\%). In 6 (5.9\%) cases with chronic bilateral lower leg skin changes, we found no documented evidence of cellulitis but antibiotics had still been prescribed for these patients. In the remaining 95 cases with infection (39 purulent and 56 nonpurulent), antibiotic selection and duration was guideline concordant in 75 (78.9\%) and $86(90.5 \%)$ cases, respectively.

The 108 ARI cases included 55 cases (50.9\%) of upper respiratory tract infections, 20 cases $(18.5 \%)$ with pharyngitis, 13 cases (12.0\%) of sinusitis, and 20 cases (18.5\%) of acute bronchitis, which included 1 case recategorized as an acute exacerbation of chronic obstructive pulmonary disease. In 76 cases (70.4\%) an antibiotic was not indicated and not prescribed. In 29 cases (26.9\%) an antibiotic was not indicated but had still been prescribed, and in 3 cases (2.8\%) an antibiotic was both indicated and prescribed.

Antibiotic recommendations were made by emergency department clinicians in 81 (25.3\%) of the manually reviewed cases; recommendations were made by internal medicine clinicians in 89 cases $(27.8 \%)$; and recommendations were made by infectious diseases clinicians in 2 cases $(0.6 \%)$. At least 1 of these services was involved in $161(50.3 \%)$ of all reviewed cases, including 54 cases 


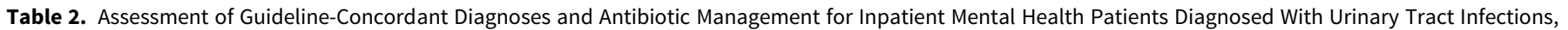
Skin and Soft-Tissue Infections, and Acute Respiratory Tract Infections Across 111 VHA Medical Centers, 2016-2018

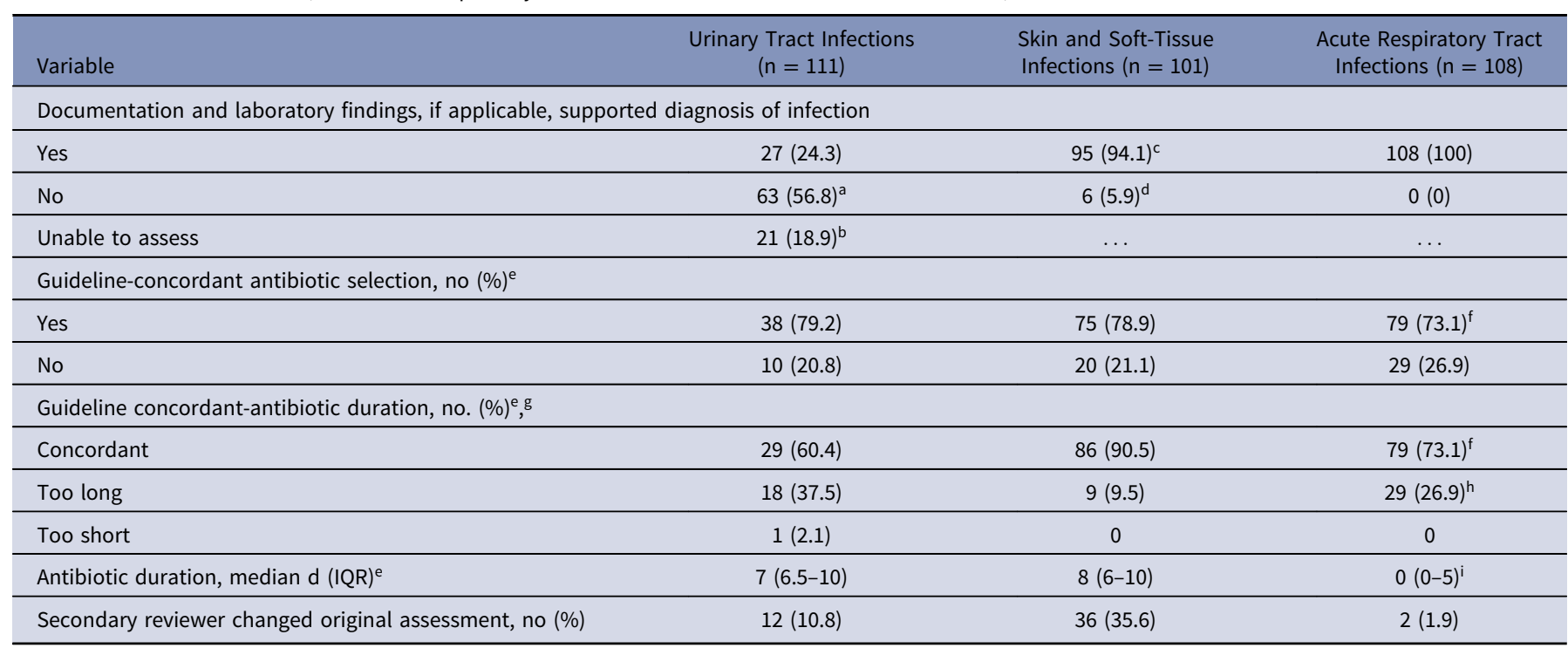

Note. IQR, interquartile range.

${ }^{a}$ There were 56 cases with the documented absence of local genitourinary symptoms (ie, asymptomatic) and an additional 7 cases had urine cultures collected that were negative before antibiotic therapy.

${ }^{b}$ In these 21 cases, documentation in the medical record did not specify whether or not the patient had local genitourinary symptoms, such as dysuria, urgency, frequency, etc.

${ }^{c}$ There were 39 purulent infections and 56 nonpurulent infections. Of the purulent infections, 21 underwent incision and drainage.

${ }^{\mathrm{d}}$ In these 6 cases, the documentation of skin findings was not suggestive of cellulitis. These were all cases with bilateral lower leg skin changes that were thought to be chronic.

'This category only includes cases in which documentation and laboratory findings, if applicable, supported the diagnosis of infection. For the diagnosis of urinary tract infections, cases deemed

"unable to assess" were also included. Antibiotic selection was assessed on day 3 of therapy.

fIn these 79 cases, an antibiotic was both indicated and prescribed in 3 cases, and in 76 cases an antibiotic was not indicated and was not prescribed.

${ }^{\mathrm{g}}$ To assess guideline-concordant duration, any duration of therapy that was $\leq$ or $\geq 2$ days from the guideline recommendation was considered concordant (Supplementary Figs. 1-7 online).

hIn these 29 patients who did not meet criteria for antibiotic therapy, the median duration of antibiotics was 5 days (IQR, 5-10).

'In the 32 patients with an ARI who were prescribed an antibiotic, the median duration of therapy was 5 days (IQR, 5-10).

(48.7\%) with UTI, 70 cases (69.3\%) with SSTI, and 37 cases $(34.3 \%)$ with ARI. When these nonpsychiatric services were involved, we detected no differences in the quality of antibiotic-prescribing for UTIs or SSTIs and less guideline-concordant management for ARI (Supplementary Table 3 online).

\section{Discussion}

In this study across 111 inpatient mental health units in the VHA, 1 of every 10 patients was exposed to an antibiotic. In contrast, studies in acute-care hospitals have reported that half of all patients are exposed to an antibiotic. ${ }^{4}$

The most common reason for antibiotic therapy in these inpatient mental health wards was a suspected UTI, but most UTI cases had the documented absence of local genitourinary symptoms or had no documentation suggesting that a clinician inquired about these symptoms. In many cases, the presence of altered mental status appeared to be a driver of antibiotic therapy even though antibiotics are not recommended for bacteriuria in the context of cognitive impairment alone. ${ }^{5}$ Overall, these findings suggest that UTI is commonly misdiagnosed and therefore represent an optimal target for stewardship efforts. We also identified some opportunities to improve antibioticprescribing for SSTIs and ARIs.

Our results revealed that emergency department and internal medicine clinicians commonly provided treatment recommendations in mental health patients with suspected infections. Because antibiotic-prescribing was not consistently better when these services were involved, antibiotic stewardship interventions to improve antibiotic prescribing in VHA mental health units must target both mental health clinicians as well as these other physician disciplines. $^{6}$

Our findings are in line with prior work that has described the frequent misdiagnosis of UTIs. ${ }^{78}$ We speculate that a major contributor to the overdiagnosis of UTIs is the seemingly routine performance of urinalyses in admitted patients. ${ }^{9}$ More thoughtful ordering and interpretation of urine studies may help mitigate incorrect UTI diagnoses and subsequent unnecessary antibiotic use. ${ }^{10}$

Our study had a few limitations. First, our manual chart reviews were dependent on the quality of the clinicians' documentation and our ability to ascertain key data elements in the medical record. To minimize the possibility of omission, we had 2 team members review each included case. Second, to assess guideline concordance, we sampled an equal number of cases from each facility regardless of bed size. As a result, antibiotic-prescribing practices at smaller facilities, which contributed less to total national antibiotic use, may have disproportionately influenced our overall assessments of guideline discordance. Third, because we specifically looked for cases coded as a UTI, we may have overlooked asymptomatic cases in which abnormal urine studies were correctly not attributed to a UTI. Finally, our findings may not be generalizable to non-VHA settings.

In conclusion, our study measured and evaluated antibioticprescribing across inpatient mental health units, which have been overlooked by both antibiotic stewardship metrics and by prior research. Future antibiotic stewardship interventions in mental health units should focus on the accurate diagnosis of UTIs while 
targeting the different physician disciplines involved in antibiotic decision making for mental health patients.

Acknowledgments. We thankk Lucas W. Watson, PharmD, BCPP, for his input on our approach to the manual chart reviews.

Financial support. This work was supported in part by a Career Development Award (DJL) from the VA Health Services Research and Development Service (CDA 16-204).

Conflict of interest. All authors report no conflicts of interest.

Supplementary material. To view supplementary material for this article, please visit https://doi.org/10.1017/ice.2021.432

\section{References}

1. National Healthcare Safety Network. Antimicrobial use and resistance module. Centers for Disease Control and Prevention website. https:// www.cdc.gov/nhsn/pdfs/pscmanual/11pscaurcurrent.pdf. Published 2021. Accessed June 10, 2021.

2. Griebel ME, Heintz B, Alexander B, Egge J, Goto M, Livorsi DJ. Understanding changes in the standardized antimicrobial administration ratio for total antimicrobial use after implementation of prospective audit and feedback. Infect Control Hosp Epidemiol 2018;39:1476-1479.
3. Livorsi DJ, Nair R, Dysangco A, et al. Using audit-and-feedback to improve antimicrobial-prescribing in emergency departments: a multicenter quasiexperimental study in the Veterans' Health Administration. Open Forum Infect Dis 2021;8(6):ofab186.

4. Magill SS, Edwards JR, Beldavs ZG, et al. Prevalence of antimicrobial use in US acute care hospitals, May-September 2011. JAMA 2014;312: $1438-1446$.

5. Nicolle LE, Gupta K, Bradley SF, et al. Clinical practice guideline for the management of asymptomatic bacteriuria: 2019 update by the Infectious Diseases Society of America. Clin Infect Dis 2019;68(10): e83-e110.

6. Petty LA, Vaughn VM, Flanders SA, et al. Assessment of testing and treatment of asymptomatic bacteriuria initiated in the emergency department. Open Forum Infect Dis 2020;7(12):ofaa537.

7. Spivak ES, Burk M, Zhang R, et al. Management of bacteriuria in Veterans' Affairs hospitals. Clin Infect Dis 2017;65:910-917.

8. Grein JD, Kahn KL, Eells SJ, et al. Treatment for positive urine cultures in hospitalized adults: a survey of prevalence and risk factors in 3 medical centers. Infect Control Hosp Epidemiol 2016;37:319-326.

9. Yin P, Kiss A, Leis JA. Urinalysis orders among patients admitted to the general medicine service. JAMA Intern Med 2015;175:1711-1713.

10. Morgan DJ, Croft LD, Deloney V, et al. Choosing wisely in healthcare epidemiology and antimicrobial stewardship. Infect Control Hosp Epidemiol 2016;37:755-760 